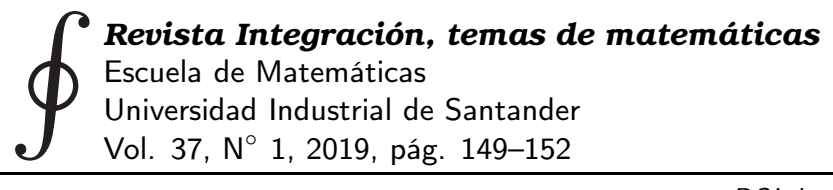

DOI: http://dx.doi.org/10.18273/revint.v37n1-2019007

\title{
Continuous images of hereditarily indecomposable continua
}

\author{
DAVID P. BELLAMY* \\ University of Delaware, Professor of Mathematical Sciences, emeritus, Newark, USA.
}

\begin{abstract}
The theorem proven here is that every compact metric continuum is a continuous image of some hereditarily indecomposable metric continuum. Keywords: Continuous maps, continuum, hereditarily indecomposable. MSC2010: 54F15, 54F45, 54E45, 54C60.

\section{Imágenes continuas de continuos hereditariamente indescomponibles}

Resumen. El teorema demostrado es que todo continuo métrico es imagen continua de algún continuo métrico hereditariamente indescomponible.

Palabras clave: Funciones continuas, continuo, hereditariamente indescomponible.

\section{Introduction}

These definitions are needed in what follows and may or may not be familiar to everyone. A continuum $X$ is a compact, connected metric space. A continuum $X$ is indecomposable provided that whenever $A$ and $B$ are proper subcontinua of $X, A \cup B$ is a proper subset of $X ; X$ is hereditarily indecomposable if, and only if, every subcontinuum of $X$ is indecomposable. A map is a continuous function. A map $f$ from a continuum $X$ to a continuum $Y$ is weakly confluent provided that given any continuum $M \subseteq Y$ there exists a continuum $W \subseteq X$ such that $f(W)=M$. When $X$ is a continuum, $C(X)$ is the hyperspace of subcontinua of $X$. If $a$ and $b$ are points in $\mathbb{R}^{n}$ with $a \neq b,[a, b]$ denotes the line segment from $a$ to $b$. Let $S^{n}$ denote the $n$ dimensional sphere. An arc $A \subseteq S^{3}$ is tame if and only if there is a homeomorphism $h: S^{3} \rightarrow S^{3}$ such that $h(A)$ is an arc of a great circle in $S^{3}$.

In [4] J. W. Rogers, Jr. asked whether every continuum is a continuous image of some indecomposable continuum. The author [1] gave an affirmative answer to this question.

\footnotetext{
${ }^{*}$ E-mail: bellamy@udel.edu

Received: 16 November 2018, Accepted: 4 January 2019.

To cite this article: D.P. Bellamy, Continuous Images of Hereditarily Indecomposable Continua, Rev. Integr. temas mat. 37 (2019), No. 1, 149-152. doi: 10.18273/revint.v37n1-2019007.
} 
Some time later, in conversation, Rogers asked whether every continuum is a continuous image of some hereditarily indecomposable continuum. This article provides a proof that the answer to this question is also yes.

The author first announced this result in [1] but has not published it previously. It has come to my attention that in [4] this result has been extended to the non-metric case, building on the metric result.

\section{Necessary Lemmas}

Lemma 2.1. Let $X$ and $Y$ be continua. Then $f: X \rightarrow Y$ is weakly confluent if, and only if, the hyperspace map induced by $f, C(f): C(X) \rightarrow C(Y)$, is surjective.

Proof. This is just a restatement of the definition of weakly confluent.

Lemma 2.2. There exists a hereditarily indecomposable subcontinuum of $\mathbb{R}^{4}$ which separates $\mathbb{R}^{4}$.

Remark on proof. R. H. Bing [2] proved this not just for $n=4$, but for every $n>1$.

Lemma 2.3. Each homotopically essential map from a continuum $X$ to the three sphere, $S^{3}$, is weakly confluent.

Proof. This was essentially proven, although in a different context, by S. Mazurkiewicz in [5, Theoreme I, p. 328]. This argument gives the necessary details. Let $X$ be a continuum, and suppose $g: X \rightarrow S^{3}$ be a homotopically essential map. To prove that $g$ is weakly confluent, it suffices to prove that every tame arc in $S^{3}$ is equal to $g(M)$ for some continuum $M \subseteq X$. This follows from Lemma 2.1 because the set of tame arcs is dense in $C\left(S^{3}\right)$.

First, set up some machinery and notation, as follows. Let $J$ be a tame arc in $S^{3}$; let $D_{n}$ be the closed disk in the complex plane with radius $(1 / n)$ centered at 0 . Let $E_{n}$ be the corresponding open disk, and let $T_{n}$ be the circle $D_{n} \backslash E_{n}$. Let $C_{n}$ be the solid cylinder $D_{n} \times[0,1]$. Since $J$ is a tame, there exists an embedding $h$ of $C_{1}$ into $S^{3}$ such that $h(\{0\} \times[0,1])=J$. Consider $C_{n}$ as a subset of $S^{3}$ by identifying $C_{1}$ with $h\left(C_{1}\right)$, and for each $t \in[0,1]$ let $t$ denote the point $h(0, t) \in J$.

Let $F_{n}$ denote the manifold boundary of $C_{n}$, that is, $F_{n}=\left(D_{n} \times\{0,1\}\right) \cup\left(T_{n} \times[0,1]\right)$. Note that given any $n$ and any $a, b \in J$ there is an isotopy $H: C_{n} \times[0,1] \rightarrow C_{n}$ satisfying the following:

(i) for each $s \in[0,1], H(J \times\{s\})=J$;

(ii) for each $x \in F_{n}$ and each $t \in[0,1], H(x, t)=x$;

(iii) for every $x \in C_{n}, H(x, 0)=x$; and

(iv) $H(b, 1)=a$. 
By setting $H(x, t)=x$ for every $x \in S^{3} \backslash C_{n}$, and every $t \in[0,1], H$ can be considered to be a function (hence an isotopy) from $S^{3} \times[0,1]$ to $S^{3}$.

Now, suppose $X$ is a continuum and let $g: X \rightarrow S^{3}$ be a homotopically essential map. To prove that $g$ is weakly confluent, it suffices to prove that there exists a continum $M \subseteq X$ such that $g(M)=J$.

Proceed by contradiction; assume there is no such $M$. Then no component of $g^{-1}(J)$ intersects both $g^{-1}(0)$ and $g^{-1}(1)$. By compactness, there is a separation, $R_{0} \cup R_{1}$ of $g^{-1}(J)$ satisfying $g^{-1}(0) \subseteq R_{0}$ and $g^{-1}(1) \subseteq R_{1}$. Since $R_{0}$ and $R_{1}$ are disjoint closed sets in $X$, there exist open subsets $S_{0}$ and $S_{1}$ of $X$ such that $R_{0} \subseteq S_{0}$ and $R_{1} \subseteq S_{1}$ and $C l\left(S_{0}\right) \cap C l\left(S_{1}\right)=\emptyset$. There exists $n$ such that $g^{-1}(C n) \subseteq S_{0} \cup S_{1}$. Let $p=\inf g\left(R_{1}\right)$ and let $q=\sup g\left(R_{0}\right)$, and let $a, b \in J$ be such that $0<a<p$ and $q<b<1$. If $p>q$, then $g$ is not surjective and hence not essential, so $0<a<p \leq q<b<1$. Using the number $n$ and the points $a$ and $b$ just chosen, let $H: S^{3} \times[0,1] \rightarrow S^{3}$ be the isotopy described above. Define a homotopy $G: X \times[0,1] \rightarrow S^{3}$ by $G(x, t)=g(x)$ if $x \in X \backslash S_{0}$ and $G(x, t)=H(g(x), t)$ if $x \in C l\left(S_{0}\right)$. Define $f: X \rightarrow S^{3}$ by $f(x)=G(x, 1)$.

Then, note that if $y \in J$ and $a<y<p$, then there does not exist $z \in X$ such that $f(z)=y$, so $f$ is nonsurjective. Hence, $f$ is inessential. Since $g$ is homotopic to $f, g$ is inessential also, a contradiction, which completes the proof.

Lemma 2.4. A continuum $X \subseteq R^{4}$ admits a homotopically essential map onto $S^{3}$ if, and only if, $R^{4} X$ is not connected $S^{3}$.

Remark on Proof. This is a special case of the Borsuk separation theorem. I do not have a reference to the original proof, but a proof can be found in almost any advanced topology or algebraic topology book.

Lemma 2.5. Given any continuum $Y$, there is a continuum $X \subseteq S^{3}$ that admits a continuous surjection $f: X \rightarrow Y$.

Proof. Let $Y$ be a continuum and let $C$ and $D$ be Cantor sets in $R^{3}$ such that $C$ and $D$ lie on lines skew to each other. Then, whenever $a, p \in C$ and $b, q \in D$, and $a, p, b$, and $q$ are all different, the line segments $[a, b]$ and $[p, q]$ are disjoint. Let $g: C \cup D \rightarrow Y$ be a map such that $g \mid C: C \rightarrow Y$ and $g \mid D: D \rightarrow Y$ are both onto. Such a $g$ exists since a Cantor set can be mapped onto every compact metric space. Define $X=\bigcup\{[a, b]: a \in$ $C ; b \in D$ and $g(a)=g(b)\}$. Then $X$ is a continuum in $R^{3}$. For each $x \in X$, let $[a(x), b(x)]$ be a segment in $X$ satisfying $a(x) \in C ; b(x) \in D$, and $x \in[a(x), b(x)]$. (This segment is unique unless $x=a(x)$ or $x=b(x)$.) Define $f: X \rightarrow Y$ by $f(x)=g(a(x))=g(b(x))$. It is straightforward to verify that $f: X \rightarrow Y$ is continuous and onto. Since for any point $p \in S^{3}, S^{3} \backslash\{p\}$ is a copy of $R^{3}, X$ can be treated as a subcontinuum of $S^{3}$.

\section{Main Result}

Theorem 3.1. Let $Y$ be an arbitrary continuum. There exists a hereditarily indecomposable continuum $K$ that admits a surjective map $f: K \rightarrow Y$.

Vol. 37, $\left.\mathrm{N}^{\circ} 1,2019\right]$ 
Proof. Let $Y$ be a continuum. By Lemma 2.5, there is a continuum $T \subseteq S^{3}$ and an onto map $g: T \rightarrow Y$. By Lemma 2.2, there exists a hereditarily indecomposable continuum $L \subseteq R^{4}$ that separates $R^{4}$. Thus by Lemma 2.4 , there is a homotopically essential map $h: L \rightarrow S^{3}$. By Lemma 2.3, $h$ is weakly confluent, so there exists a continuum $K \subseteq L$ such that $h(K)=T$. Let $f=g \circ(h \mid K)$. Then $f: K \rightarrow Y$ is the desired map; $K$ is hereditarily indecomposable since it is a subcontinuum of $L$.

\section{References}

[1] Bellamy D.P., "Continuous mappings between continua", in Topology Conference Guilford College, 1979, Guilford College (1980), 101-112.

[2] Bellamy D.P., "Mappings of indecomposable continua", Proc. Amer. Math. Soc. 30 (1971), 179-180.

[3] Bing R.H., "Higher-dimensional hereditarily indecomposable continua", Trans. Amer. Math. Soc. 71 (1951), 267-273.

[4] Hart K.P., van Mill J. and Pol R., "Remarks on hereditarily indecomposable continua", https://arXiv.org/pdf/math/0010234.pdf

[5] Mazurkiewicz S., "Sur l'existence des continus indécomposables", Fund. Math. 25 (1935), No. 1, 327-328.

[6] Rogers J.W.Jr., "Continuous mappings on continua", Proc. Auburn Topology Conference, Auburn University, Auburn, USA, 1969, 94-97. 